\title{
ON AN EXISTENCE RESULT FOR NONLINEAR EVOLUTION INCLUSIONS
}

\author{
by STANISLAW MIGÓRSKI
}

(Received 13th July 1994)

In this paper we present an existence result for a class of nonlinear evolutions inclusions. A result on the compactness of the solution set for a differential inclusion is also established.

1991 Mathematics subject classification: 34G20.

\section{Introduction}

Recently, in an interesting paper [10], Papageorgiou has studied the dependence of the solutions of an evolution inclusion of the form

$$
\left\{\begin{array}{l}
\dot{x}(t)+A(t, x(t)) \in F(t, x(t)) \text { a.e. } t \in(0, T) \\
x(0)=x_{0}
\end{array}\right.
$$

on the parameter. However, the main existence theorem of his paper (see Theorem 3.1 in [10]) ensuring the nonemptiness of the solution set for the evolution inclusion (1) has a gap in its proof and therefore, in our opinion, is incorrect. Namely, Papageorgiou (see [10, pp. 145-146]) exploited in his proof the result of Nagy [6, Theorem 2] which states that $\mathscr{W}$ (the space, where the solution of the inclusion is sought) is compactly embedded into $C(0, T ; H)$ (the space of continuous functions from the time interval $[0, T]$ into $H$ ); see the notation of Section 2 below. In [5] the present author has delivered an example which shows that Theorem 2 of Nagy [6] is false. For this reason, the proof of existence of solutions to (1) can not arise directly from the reasoning given in [10].

We remark that in the paper [12], Papageorgiou has extensively used the existence theorem ([10, Theorem 3.1]) and the result of Nagy ([6, Theorem 2]) to get the existence of optimal trajectories in some variational problems associated with evolution inclusion (1).

It can be observed that the results of [10] and [12] on the compactness of the solution set of $(1)$ in $C(0, T ; H)$ are valid without an appeal to Nagy's inaccurate result and for $V$ a separable Banach space, provided that $x_{0} \in V$. In fact, in this case the solution set of (1) is sequentially compact in $C(0, T ; w-V)$ (see [1] or [8]) and hence Corollary 4, p. 85 of Simon [13] entails that it is compact in $C(0, T ; H)$.

In this paper we are going to present the result on existence of solutions to the 
evolution differential inclusion (1) with the initial condition $x_{0} \in H$. We follow the procedure that closely resembles the one of Papageorgiou [10]. A crucial point in our approach is the fact that the solution map of the associated evolution equation is continuous in suitable topologies (see Proposition 1). Let us underline that in this way, we obtain the existence result, given previously in [10], under weaker hypothesis that $V$ (the space appearing in the Gelfand triple, see Section 2) is a reflexive Banach space and not a Hilbert one as it was requried in [10]. This allows us to enlarge the class of problems to which the result can be applied. Proposition 1 and Corollary 1 given in Section 3 show that Theorem 3.2 of [12] is true (even for $x_{0} \in H$ ) and the results concerning variational problems (see Theorem 4.3 and Theorem 4.4 in [12]) are still valuable. Finally, we mention that Theorem 1 (for $p=q=2)$ can be found also in [9] and a "nonconvex" version of it was proved in Theorem 3.4 of [11].

For the convenience of the reader, we firstly present the result on the continuous dependence of solutions to the evolution equation on the right hand side and then we give the proof of the existence result indicating the place into where the new ingredient comes.

\section{Preliminaries}

Let $H$ be a separable Hilbert space and let $V$ be a subspace of $H$ carrying the structure of a separable reflexive Banach space, which embeds into $H$ densely and continuously. Identifying $H$ with its dual, we have the Gelfand triple (see e.g. [15]) $V \subset H \subset V^{\prime}$, where all embeddings are continuous and dense. Moreover, we assume in this paper that these embeddings are also compact. We denote by $\langle\cdot, \cdot\rangle$ the duality of $V$ and its dual $V^{\prime}$ as well as the inner product on $H$, by $\|\|,.|$.$| and \|.\|_{V^{\prime}}$ the norms in $V, H$ and $V^{\prime}$, respectively. Given a fixed real number $T>0$ and $2 \leqq p<+\infty$, we introduce the following spaces $\mathscr{V}=L^{p}(0, T ; V), \quad \mathscr{H}=L^{p}(0, T ; H), \quad \mathscr{H}^{\prime}=L^{q}(0, T ; H), \quad \mathscr{V}^{\prime}=L^{q}\left(0, T ; V^{\prime}\right)$, $(1 / p+1 / q=1)$ and $\mathscr{W}=\left\{w \in \mathscr{V} \mid w^{\prime} \in \mathscr{V}^{\prime}\right\}$, where the derivative is understood in the sense of vector valued distributions. Clearly $\mathscr{W} \subset \mathscr{V} \subset \mathscr{H} \subset \mathscr{V}^{\prime}$. The pairing of $\mathscr{V}$ and $\mathscr{V}^{\prime}$ and the duality between $\mathscr{H}$ and $\mathscr{H}^{\prime}$ are denoted by

$$
\langle\langle f, v\rangle\rangle=\int_{0}^{T}\langle f(s), v(s)\rangle d s .
$$

Given a Banach space $\mathscr{X}$, the symbols $w-\mathscr{X}, s-\mathscr{X}$ are always used to indicate the space $\mathscr{X}$ equipped with the weak and the strong (norm) topology, respectively.

Let $(\Omega, \Sigma, \mu)$ be a measure space, $X$ be a separable Banach space. A multifunction $F$ defined on $\Omega$ with values in the space $2^{X}$ of all nonempty subsets of $X$ is called measurable if $F^{-}(E):=\{\omega \in \Omega: F(\omega) \cap E \neq \emptyset\} \in \Sigma$, for every closed set $E \subset X . F$ is called graph measurable if $G r F:=\{(\omega, x) \in \Omega \times X: x \in F(\omega)\} \in \Sigma \times \mathscr{B}(X)$ (here $\mathscr{B}(X)$ is the family of all Borel subsets of $X)$. We denote by $S_{F}^{p}(1 \leqq p \leqq \infty)$ the set of all selectors of $F$ that belong to $L^{p}(\Omega ; X)$ i.e. $S_{F}^{p}=\left\{f \in L^{p}(\Omega ; X): f(\omega) \in F(\omega) \mu\right.$ a.e. $\}$. Let $\left(Y, \tau_{Y}\right),\left(Z, \tau_{Z}\right)$ be Hausdorff topological spaces. A multifunction $G: Y \rightarrow 2^{Z}$ is $\left(\tau_{Y}-\tau_{Z}\right)$ upper semi- 
continuous (u.s.c.), if for every $C \subset Z$ closed in $\tau_{Z}$ topology, $G^{-}(C)$ is closed in $\tau_{Y}$ topology in $Y$. By $\mathscr{P}_{f(c)}(X)$ we denote the family of all nonempty, closed, (convex) subsets of $X$. Finally, given a sequence of sets $S_{n} \in 2^{Y}$, the sequential Kuratowski upper limit is defined by

$$
\tau_{Y}-\limsup S_{n}=\left\{y \in Y: \exists\left\{n_{v}\right\}, y_{n_{r}} \in S_{n_{r}}, y_{n_{r}} \stackrel{\tau x}{\longrightarrow} y \text {, as } v \rightarrow+\infty\right\}
$$

\section{Existence theorem}

Definition. A function $x \in \mathscr{W}$ is called a solution of problem (1) if and only if

$$
\left\{\begin{array}{l}
\dot{x}(t)+A(t, x(t))=f(t) \text { a.e. } t \in(0, T) \text { in } V^{\prime} \\
x(0)=x_{0}
\end{array}\right.
$$

with $f \in S_{F(\cdot, x(\cdot))}^{q}$.

We will need the following hypotheses:

$H(A): \quad A:[0, T] \times V \rightarrow V^{\prime}$ is an operator such that

(1) $t \rightarrow A(t, v)$ is measurable from $[0, T]$ to $V^{\prime}$,

(2) $v \rightarrow A(t, v)$ is hemicontinuous and monotone from $V$ to $V^{\prime}$,

(3) $\|A(t, v)\|_{V^{\prime}} \leqq a\left(1+\|v\|^{p-1}\right)$, a.e. $t \in(0, T), \forall v \in V$ with $a>0$,

(4) $\langle A(t, v), v\rangle \geqq c\|v\| p$, a.e. $t \in(0, T)$ with $c>0$.

$H(F): \quad F:[0, T] \times H \rightarrow \mathscr{P}_{f c}(H)$ is a multifunction such that

(1) $(t, x) \rightarrow F(t, x)$ is graph measurable,

(2) $G r F(t, \cdot)$ is sequentially closed in $H \times(w-H)$, a.e. $t \in(0, T)$,

(3) $|F(t, x)| \leqq a(t)+b|x|^{2 / q}$, a.e. $t \in(0, T)$, with $a \in L_{+}^{q}(0, T), b>0$.

The hypotheses $H(A)$ and $H(F)$ for $p=q=2$ coincide with the ones of Papageorgiou [10].

The following result concerns the solution map for (2) and plays a crucial rôle in the proof of the main theorem.

Proposition 1. If hypothesis $H(A)$ holds and $x_{0} \in H$, then the map which to every right hand side $f \in \mathscr{H}^{\prime}$ in (2) assigns the unique solution $x=x(f) \in \mathscr{W} \subset C(0, T ; H)$ of (2), is continuous from $w-\mathscr{H}^{\prime}$ into $w-\mathscr{W}$ and from $w-\mathscr{H}^{\prime}$ into $C(0, T ; H)$.

Proof. Let $f_{n}, f \in \mathscr{H}^{\prime}$ be such that

$$
f_{n} \rightarrow f \text { in } w-\mathscr{H}^{\prime}
$$


and let $x_{n}=x\left(f_{n}\right)$ be the sequence of unique solutions to (2) in $\mathscr{W}$ corresponding to $f_{n}$ (see Theorem 4.2 of Barbu [3, p. 167] or Theorem 1.2 of Lions [4, p. 162]). From the classical a priori estimates on the solutions to parabolic equations (see Chapter 2.1 of Lions [4]), we know that $\left\{x_{n}\right\}$ lies in a bounded subset of $\mathscr{W}$. By passing to a subsequence, if necessary, we may assume that

$$
x_{n} \rightarrow x \text { in } w-\mathscr{W},
$$

for some $x \in \mathscr{W}$. Since $\mathscr{W} \subset \mathscr{H}$ compactly (see Theorem 5.1 in [4, p. 48]), we also have

$$
x_{n} \rightarrow x \text { in } s-\mathscr{H} .
$$

Let us consider the convex set $\mathscr{W}_{x_{0}}=\left\{w \in \mathscr{W}: w(0)=x_{0}\right\}$. It is closed (and hence weakly closed) in $\mathscr{W}$, in virtue of the fact that $\mathscr{W} \subset C(0, T ; H)$ continuously (see e.g. Ahmed and Teo [1]).

Since $x_{n} \in \mathscr{W}_{x_{0}}$, we deduce from (4) that

$$
x(0)=x_{0}
$$

We claim that

$$
\underset{n}{\limsup }\left\langle\left\langle\hat{A}\left(x_{n}\right), x_{n}-x\right\rangle\right\rangle \leqq 0,
$$

where we denote by $\hat{A}: \mathscr{V} \rightarrow \mathscr{V}^{\prime}$ the Nemytski operator corresponding to $A$ i.e. $(\hat{A} v)(t)=A(t, v(t))$ for a.e. $t$. Multiplying the equation $\dot{x}_{n}+\hat{A}\left(x_{n}\right)=f_{n}$ in duality with $x_{n}-x$, using the integration by parts formula (see e.g. Proposition 23.23, p. 422 of Zeidler [15]) and (6), we obtain

$$
\left\langle\left\langle\dot{x}, x_{n}-x\right\rangle\right\rangle+\frac{1}{2}\left|x_{n}(T)-x(T)\right|^{2}+\left\langle\left\langle\hat{A}\left(x_{n}\right), x_{n}-x\right\rangle\right\rangle=\left\langle\left\langle f_{n}, x_{n}-x\right\rangle\right\rangle .
$$

From (3) and (4), by taking the limit in (8) and dropping the positive term, we get

$$
\limsup _{n}\left\langle\left\langle\hat{A}\left(x_{n}\right), x_{n}-x\right\rangle\right\rangle \leqq \lim _{n}\left(\left\langle\left\langle f_{n}, x_{n}-x\right\rangle\right\rangle-\left\langle\left\langle\dot{x}, x_{n}-x\right\rangle\right\rangle\right)=0 .
$$

This proves the claim.

We are going to show now that by possibly reindexing and/or dropping to a further subsequence, we may assume that $x_{n} \rightarrow x$ in $C(0, T ; H)$. For every $n$ and a.e. $t \in(0, T)$, we put

$$
\lambda_{n}(t)=\left\langle A\left(t, x_{n}(t)\right)-A(t, x(t)), x_{n}(t)-x(t)\right\rangle .
$$

Applying Fatou's Lemma to a nonnegative functions $\lambda_{n}$ (compare hypothesis $H(A)(2)$ ), using (4) and (7), we have 


$$
\begin{gathered}
0 \leqq \int_{0}^{T} \liminf _{n} \lambda_{n}(s) d s \leqq \liminf _{n} \int_{0}^{T} \lambda_{n}(s) d s \leqq \sup _{n} \int_{0}^{T} \lambda_{n}(s) d s \\
\leqq \limsup _{n} \int_{0}^{T}\left\langle A\left(s, x_{n}(s)\right), x_{n}(s)-x(s)\right\rangle d s-\liminf _{n} \int_{0}^{T}\left\langle A(s, x(s)), x_{n}(s)-x(s)\right\rangle d s \\
=\limsup \left\langle\left\langle\hat{A}\left(x_{n}\right), x_{n}-x\right\rangle\right\rangle \leqq 0 .
\end{gathered}
$$

From the above inequalities, we deduce that $\lim _{n} \int_{0}^{T} \lambda_{n}(s) d s=0$, which thanks to the nonnegativity of $\lambda_{n}$, implies $\lambda_{n} \rightarrow 0$ strongly in $L^{1}(0, T)$. Therefore, we may assume, passing to a next subsequence, that

$$
\lambda_{n}(t) \rightarrow 0 \text { a.e. } t \in(0, T)
$$

Using hypothesis $H(A)(3)(4)$, for a.e. $t \in(0, T)$, we have

$$
\begin{aligned}
& \lambda_{n}(t)=\left\langle A\left(t, x_{n}(t)\right), x_{n}(t)\right\rangle-\left\langle A\left(t, x_{n}(t)\right), x(t)\right\rangle-\left\langle A(t, x(t)), x_{n}(t)\right\rangle+\langle A(t, x(t)), x(t)\rangle \\
& \quad \geqq c\left\|x_{n}(t)\right\|^{p}-a\|x(t)\|\left\|x_{n}(t)\right\|^{p-1}-a\left(1+\|x(t)\|^{p-1}\right)\left\|x_{n}(t)\right\|-a\|x(t)\|+c\|x(t)\| p .
\end{aligned}
$$

From this inequality and (9), it follows that $\left\{\left\|x_{n}(t)\right\|\right\}$ is bounded for a.e. $t \in(0, T)$ and $n \geqq n_{0}$. So we have shown that the sequence $\left\{x_{n}\right\}$ belongs to a bounded set of $L^{\infty}(0, T ; V)$. Moreover, since the sequence $\left\{\dot{x}_{n}\right\}$ lies in a bounded subset of $\mathscr{V}^{\prime}$ and $V \subset H$ compactly, we deduce, by a version of the Arzelá-Ascoli theorem (compare Corollary $4, \S 8$ in [13]) that

$$
x_{n} \rightarrow x \text { in } C(0, T ; H) \text {. }
$$

Now, it remains to show that $x$ is a solution of (2). From hypothesis $H(A)(2)$, it follows (see Proposition 2.5 in Chapter 2 of Lions [4]) that $\hat{A}$ has the generalized pseudomonotone property $(M)$ (i.e. $v_{n} \rightarrow v$ in $w-\mathscr{V}, \hat{A} v_{n} \rightarrow \chi$ in $w-\mathscr{V}^{\prime}$ and $\lim \sup _{n}\left\langle\left\langle\hat{A} v_{n}, v_{n}-v\right\rangle\right\rangle \leqq 0$ imply $\left.\chi=\hat{A} v\right)$. In view of hypothesis $H(A)(3),\left\{\hat{A} x_{n}\right\}$ is bounded in $\mathscr{V}^{\prime}$, so we may assume that

$$
\hat{A}\left(x_{n}\right) \rightarrow z \quad \text { in } w-\mathscr{V}^{\prime}
$$

with $z \in \mathscr{V}^{\prime}$. From (4), (7) and (10), we have $z=\hat{A} x$ and $\hat{A} x_{n} \rightarrow \hat{A} x$ in $w-\mathscr{V}^{\prime}$. We pass to the limit in the equation

$$
\left\langle\left\langle\dot{x}_{n}, \Psi\right\rangle\right\rangle+\left\langle\left\langle\hat{A}\left(x_{n}\right), \Psi\right\rangle\right\rangle+\left\langle\left\langle f_{n}, \Psi\right\rangle\right\rangle, \quad \forall \Psi \in \mathscr{V}
$$

and we get $\langle\langle\dot{x}, \Psi\rangle\rangle+\langle\langle\hat{A}(x), \Psi\rangle\rangle+\langle\langle f, \Psi\rangle\rangle$ for every $\Psi \in \mathscr{V}$. Hence

$$
\dot{x}(t)+A(t, x(t))=f(t) \quad \text { a.e. } \quad t \in(0, T),
$$


which together with (6) implies that $x=x(f)$ is a solution to (2). From the uniqueness of solutions of (2), we infer that the whole sequence $\left\{x_{n}\right\}$ converges to $x$ in both $w-\mathscr{W}$ and $C(0, T ; H)$. This completes the proof of the proposition.

Theorem 1. If hypotheses $H(A), H(F)$ hold and $x_{0} \in H$, then (1) admits a solution.

Proof. We follow the ideas used in [10] for the case $p=q=2$.

Step 1. We will show a priori estimates on a solution. Suppose that $x \in \mathscr{W}$ is a solution of (1). We multiply in duality the first equation in (2) by $x(\cdot)$ and using Schwarz inequality, we obtain

$$
|x(t)|^{2}+2 c \int_{0}^{t}\|x(s)\|^{p} d s \leqq\left|x_{0}\right|^{2}+2 \int_{0}^{t}|f(s)||x(s)| d s
$$

for every $t \in[0, T]$. Using Young's inequality

$$
a b \leqq \frac{\varepsilon^{p}}{p} a^{p}+\frac{\varepsilon^{-q}}{q} b^{q}, \forall a, b, \varepsilon>0, p>1,1 / p+1 / q=1
$$

and the fact that $H(F)(3)$ implies

$$
|f(s)|^{q} \leqq 2^{q-1}\left((a(s))^{q}+b^{q}|x(s)|^{2}\right),
$$

we easily get

$$
\begin{aligned}
|x(t)|^{2} & \left.+2 c \int_{0}^{t}\|x(s)\|^{p} d s \leqq\left|x_{0}\right|^{2}+\frac{2 \varepsilon^{p} \beta^{p}}{p} \int_{0}^{t} \| x(s)\right) \|^{p} d s \\
& +\frac{2^{q}}{q \varepsilon^{q}} \int_{0}^{t}(a(s))^{q} d s+\frac{1}{q}\left(\frac{2 b}{\varepsilon}\right)^{q} \int_{0}^{t}|x(s)|^{2} d s
\end{aligned}
$$

where $\beta>0$ is such that $|\cdot| \leqq \beta\|\cdot\|$. It exists since by hypothesis $V \subset H$ continuously. Choosing $\varepsilon=\frac{1}{\beta}(p c)^{1 / p}$, the Gronwall lemma implies

$$
|x(t)| \leqq M, \quad M>0
$$

Next, we also have

$$
\left.2 c \int_{0}^{t} \| x(s)\right)\|\|^{p} d s \leqq\left|x_{0}\right|^{2}+\frac{2 \varepsilon^{p}}{p} M^{p} T+\frac{2^{q}}{q \varepsilon^{q}}\|a\|_{L^{q}}+\frac{1}{q}\left(\frac{2 b}{\varepsilon}\right)^{q} M^{2} T,
$$


for every $t \in[0, T]$. This implies that

$$
\|x\|_{r} \leqq M_{1}, \quad M_{1}>0 .
$$

Since

$$
\|\dot{x}(s)\|_{V^{\cdot}} \leqq\|f(s)\|_{V^{\prime}}+\|A(s, x(s))\|_{V^{\cdot}} \leqq \gamma|f(s)|+a\left(1+\|x(s)\|^{p-1}\right),
$$

with some $\gamma>0$, using (11), we have

$$
\begin{gathered}
\|\dot{x}\|_{q^{q}}=\int_{0}^{T}\|\dot{x}(s)\| q, d s \leqq 2^{q-1} \gamma^{q}\left(\|a\|_{L^{q}}+b^{q} \int_{0}^{T}|x(s)|^{2} d s\right) \\
+a^{q} T+a^{q} \int_{0}^{T}\|x(s)\|^{p} d s \leqq 2^{q-1} \gamma^{q}\left(\|a\|_{L^{q}}+b^{q} M^{2} T\right)+a^{q} T+a^{q} M_{1}^{p},
\end{gathered}
$$

which means that

$$
\|\dot{x}\|_{r^{\prime}} \leqq M_{2}, \quad M_{2}>0 .
$$

Thus it follows from (13) and (14) that there exists a finite positive number $M_{3}$ such that $\|x\|_{\mathscr{W}} \leqq M_{3}$, where $x$ is any solution to (1).

Step 2. We consider the problem (1) with a modified multivalued term. We define the modified multifunction $\hat{F}:[0, T] \times H \rightarrow \mathscr{P}_{s c}(H)$ by

$$
\hat{F}(t, x)= \begin{cases}F(t, x) & \text { if }|x| \leqq M, \\ F\left(t, \frac{M x}{|x|}\right) & \text { if }|x|>M .\end{cases}
$$

It is easy to see that $\hat{F}$ satisfies $H(F)(1)(2)$ and $|\hat{F}(t, x)| \leqq a(t)+b M^{2 / q}=: \tilde{a}(t)$, a.e. $t$, where $\tilde{a} \in L_{+}^{q}(0, T)$.

We define

$$
B=\left\{f \in \mathscr{H}^{\prime}:|f(t)| \leqq \tilde{a}(t) \text { a.e. } t\right\} \subset \mathscr{H}^{\prime}
$$

and a map $r: B \rightarrow \mathscr{W}$ given by $r(f)=x$, where $x \in \mathscr{W}$ is the unique solution to the problem (2). Let $R$ be a multifunction defined on $B$ by

$$
R(f)=S_{\hat{F}(\cdot, r(f)(\cdot))}^{1}:=\left\{f \in L^{1}(0, T ; H): f(t) \in \hat{F}(t, r(f)(t)) \text { a.e. } t\right\} .
$$

Firstly, since $\hat{F}$ is graph measurable and $L^{1}$ integrably bounded, using Aumann's selection theorem (see Theorem 5.10 in [14]), we have that $R(f) \neq \emptyset$. Moreover, since $\hat{F}$ is $\mathscr{P}_{f c}(H)$-valued and $|\hat{F}(t, r(f)(t))| \leqq \tilde{a}(t)$ a.e. $t$, the multifunction $R$ has closed and convex values and $R: B \rightarrow \mathscr{P}_{f c}(B)$. 
We will show that $R$ is $\left(w-\mathscr{H}^{\prime}\right) \times\left(w-\mathscr{H}^{\prime}\right)$ u.s.c. on $B$. Since $B$ is a compact in $w-\mathscr{H}^{\prime}$, it suffices to prove that $G r R$ is weakly-weakly closed in $B \times B$ (see Chapter $I .1$ of Aubin and Cellina [2]). Let $\left(f_{n}, z_{n}\right) \in G r R, f_{n} \rightarrow f$ and $z_{n} \rightarrow z$ both in $w-\mathscr{H}^{\prime}$. From Proposition 1, we know that

$$
r\left(f_{n}\right)(t) \rightarrow r(f)(t) \quad \text { in } \quad s-H, \forall t \in[0, T]
$$

Since $\hat{F}$ satisfies $H(F)(1)(2)$, we deduce that

$$
w-\limsup _{n} \hat{F}\left(t, r\left(f_{n}\right)(t)\right) \subset \hat{F}(t, r(f)(t))
$$

for a.e. $t$. Invoking Theorem 4.2 of [7], we have

$$
w-\limsup _{n} R\left(f_{n}\right)=w-\limsup _{n} S_{\vec{F}\left(\cdot, r\left(f_{n}\right)(\cdot)\right)}^{1} \subseteq S_{w-\limsup }^{1} \hat{F}\left(\cdot, r\left(f_{n}\right)(\cdot)\right) \subseteq S_{\hat{F}(\cdot, r(f)(\cdot))}^{1}=R(f) .
$$

The above inclusion implies that $(f, z) \in G r R$. This shows that $G r R$ is $w-w$ closed and proves that $R$ is $w-w$ u.s.c. on $B$.

Step. 3. We apply the well known Kakutani-KyFan fixed point theorem for set-valued mappings (see Chapter I.12 in [2]) to the multifunction $R$. We deduce that there exists $f^{*} \in B$ such that $f^{*} \in R\left(f^{*}\right)$. The corresponding solution $x^{*}=r\left(f^{*}\right)$ is a solution to the problem (1) with the modified multifunction $\hat{F}$. However, the same estimate as in Step 1 (see (12)), implies that $\left|x^{*}(t)\right| \leqq M$. Thus $\hat{F}\left(t, x^{*}(t)\right)=F\left(t, x^{*}(t)\right)$ for a.e. $t$, which means that $x^{*}$ is a solution to (1). This completes the proof of the theorem.

Corollary 1. If the hypotheses of Theorem 1 hold, then the solution set of (1) is a nonempty, weakly compact subset of $\mathscr{W}$ and a compact subset of $C(0, T ; H)$.

This conclusion was earlier obtained in Theorem 3.2 of [12] on the basis of the compactness result of Nagy and under the additional assumption that $V$ is a Hilbert space.

Acknowledgement. The author wishes to thank the referee for bringing to his attention some additional references.

\section{REFERENCES}

1. N. U. Анмед and K. L. Teo, Optimal Control of Distributed Parameter Systems (NorthHolland, New York, New York, 1981).

2. J. P. Aubin and A. Cellina, Differential Inclusions (Springer-Verlag, Berlin-Heidelberg-New York-Tokyo, 1984).

3. V. Barbu, Nonlinear Semigroups and Differential Equations in Banach Spaces (Noordhoff International Publishing, Leyden, The Netherlands, 1976). 
4. J. L. Lıons, Quelques méthodes de résolution des problémes aux limites non linéaires (Dunod, Paris, 1969).

5. S. Migórski, A counterexample to a compact embedding theorem for functions with values in a Hilbert space, Proc. Amer. Math. Soc. 123 (1995), 2447-2450.

6. E. V. NAGY, A theorem on compact embedding for functions with values in an infinite dimensional Hilbert space, Ann. Univ. Sci. Budapest. Eötvös Sect. Math. 22-23 (1979-80), 243-245.

7. N. S. Papageorgiou, Convergence theorems for Banach space values integrable multifunctions, Internat. J. Math. Math. Sci. 10 (1987), 433-442.

8. N. S. Papageorgiou, Optimal control of nonlinear evolution inclusions, J. Optim. Theory Appl. 67 (1990), 321-354.

9. N. S. PAPAGEORGiou, A minimax optimal control problem for evolution inclusions, Yokohama Math. J. 39 (1991), 1-19.

10. N. S. Papageorgiou, Continuous dependence results for a class of evolution inclusions, Proc. Edinburgh Math. Soc. 35 (1992), 139-158.

11. N. S. Papageorgiou, On the set of solutions of a class of nonlinear evolution inclusions, Kodai Math. J. 15 (1992), 387-402.

12. N. S. Papageorgiou, Existence and variational problems for nonlinear evolution inclusions, Math. Japon. 38 (1993), 433-443.

13. J. Simon, Compact sets in the space $L^{p}(0, T ; B)$, Ann. Mat. Pura Appl. (4) 146 (1987), 65-96.

14. D. Wagner, Survey of measurable selection theorems, SIAM J. Control Optim. 15 (1977), 859-903.

15. E. Zeidler, Nonlinear Functional Analysis and its Applications II (Springer, New York, 1990).

INSTITUTE FOR INFORMATION SCIENCES

JAGELLONIAN UNIVERSITY

UL. NAWOJKI 11

30072 Cracow

Poland 